\title{
用有限单元法研究曾侯乙编钟双音激发过程
}

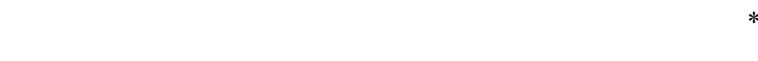 \\ (1) 中国科学院研究生院, 北京 100049; (2) 中国科学院地质与地球物理所, 北京 100029.* 联系人, E-mail: shiyl@gucas.ac.cn)
}

\begin{abstract}
摘要 曾侯乙编钟是我国宝贵的文物, 它具有敲击不同部位时会发出不同音调声音的双音特性. 从弹 性波动理论基础出发, 采用有限单元数值方法, 模拟曾侯乙编钟具有扁圆截面特殊几何形状下, 以不同 方式敲击时, 弹性波传播和自由振荡激发的过程. 通过对数值模拟激发振荡峰值分布结果的观察和对 钟体的频谱分析, 认识曾侯乙编钟双音激发物理过程的特征.
\end{abstract}

关键词 曾侯乙编钟 双音特性 有限单元法 自由振荡 波动方程

曾侯乙编钟的出土是我国考古史上的重大发现. 它在地下沉睡了 2400 多年, 是迄今为止出土数量最 多、重量最重、音律最全、气势最宏伟的一套古代编 钟，堪称“编钟之王”，是大型的国宝级文物之一.

曾侯乙编钟带给我们的不仅仅是美妙的音乐, 也带给了我们很多难解之谜. 青铜编钟是古代一种 很重要的合奏乐器, 曾侯乙编钟经过刻意设计, 与一 般钟横截面为圆形不同, 它的横截面为扁形, 敲击方 式不同时能发出 2 个不同的音调. 一般认为由于钟的 正面与侧面, 其内距不对称, 因此一个独立的编钟单 位有 2 个基频, 可以得到通常为小三度或大三度音程 的 2 个乐音 $[1.2]$, 使人能够听到高低不同的 2 种声音, 这就是编钟的双音特性.

几十年来, 很多音乐、考古、声学、物理学、冶 金及铸造等方面的专家和学者对该编钟的双音特性 进行了大量的研究工作. 他们大多数都是基于对钟 体本身的结构和特性进行物理研究, 例如华觉明 [3] 分析了青铜编钟的复杂钟腔结构和发声机制; 冯光 生 [4使用原生双音、铸生双音、铸调双音 3 个概念, 从 双音技术的角度, 对双音钟的界定、双音技术的形成 和应用范围等问题进行了讨论; 韩宝强 [2]依据多年 对编钟音响测量分析的实践经验, 提出编钟音乐性 能的判定方法, 旨在推进编钟音乐性能研究和检测 手段的现代化; 严燕来等人 “基于圆柱形模型中弹 性驻波的理论振型和椭圆形柱面的刚性和曲率分布特 征, 对古编钟的一钟双音和短延音等声学特性进行 了分析, 并分析了钟枚的长短对声音的影响作用; 王 大钧等人 ${ }^{[5]}$ 用有限单元法分析了可能的振型及形成 双音的基础.
对于编钟固有频率的各种振型的讨论 ${ }^{[1,4]}$ 虽然能 解释产生双音发音的基础, 但仅仅从固有频率出发, 无法认识曾侯乙编钟因敲击位置不同而激发双音的 物理过程. 我们目前尚未见到直接以解析方法或数 值模拟分析来研究编钟双音激发的物理过程的工作. 本文将以有限元数值方法, 模拟敲击编钟的不同部 位时弹性波的传播及激发钟体振荡的全过程, 并对 敲击不同部位激发产生的波动进行频谱分析，以便 进一步认识编钟双音激发产生的机理.

\section{1 模拟编钟的有限元模型和计算方法}

如前所述, 本文将用有限单元法模拟敲击钟体 不同部位后弹性波在钟体内的传播、反射、叠加, 最 后激发钟体振荡的过程. 在解决波动问题的产生和 传播问题上, 有限单元法具有很大的优越性 ${ }^{[6-11]}$, 它 不受求解区域的几何形状限制, 也不需要特殊的边 界条件处理. 但为了精确模拟钟体的几何形状和钟 体内波的传播, 空间上将需要超过百万至千万的三 维有限元网格, 求解时每个时间步的步长也不能过 大, 因此数值模拟计算量非常大, 对计算的算法和软 件实现提出了很高的要求.

本文的问题是求解编钟这样一个扁圆体几何模 型的波动问题，依据弹性介质的波动方程 ${ }^{[11 ~ 14]}$ :

$$
\rho \ddot{u}+c \dot{u}-(\mu \nabla \cdot(\nabla u)+(\lambda+\mu) \nabla(\nabla \cdot u))+f=0,
$$

其中 $\rho$ 为介质密度; $\ddot{u}_{i}(i=1,2,3)$ 为 3 个方向上的加 速度分量, $\dot{u}_{i}$ 为速度, $u_{i}$ 为位移; $f_{i}$ 为体力分量; $c$ 为 与介质的品质因子成反比的一个系数, 考虑钟的震 荡终将衰减, 因此包含此阻尼项. (1)式中的应力应变 关系满足虎克定律: 


$$
\sigma_{i j}=C_{i j k l} \varepsilon_{k l}, \quad(i, j, k, l=1,2,3),
$$

式中, $C_{i j k l}$ 为弹性系数, 与介质的物性有关; $\varepsilon_{i j}$ 为体 应变; $\sigma_{i j}$ 为体应力分量.

应变和位移之间的几何关系为

$$
\varepsilon_{k l}=\frac{1}{2}\left(\frac{\partial u_{l}}{\partial x_{k}}+\frac{\partial u_{k}}{\partial x_{l}}\right),(k, l=1,2,3) .
$$

对于各向同性介质, 弹性模量可以简化为 2 个独 立的弹性参数, 即 Lame 常数 $\lambda$ 和 $\mu$, 其与 $C_{i j k l}$ 关系为

$$
C_{i j k l}=\lambda \delta_{i j} \delta_{k l}+\mu\left(\delta_{i k} \delta_{j l}+\delta_{i l} \delta_{j k}\right),
$$

其中 $\delta_{i j}$ 为 Kronecker 记号.

对(1)式采用基于 Galerkin 方法的有限元求解方 法，进行空间离散化后，写成矩阵形式可得

$$
M A+C V+S U=F,
$$

其中 $M$ 为质量矩阵, $A$ 为加速度向量, $V$ 为速度向量, $U$ 为位移向量, $C$ 为阻尼矩阵, $S$ 为刚度矩阵, $F$ 为载 荷向量.

我们采用速度法的时间离散格式:

$$
\boldsymbol{M} \boldsymbol{A}^{t+\Delta t / 2}+\boldsymbol{C} \boldsymbol{V}^{t+\Delta t / 2}+\boldsymbol{S} \boldsymbol{U}^{t+\Delta t / 2}=\boldsymbol{F}^{t+\Delta t / 2},
$$

由

$$
\begin{gathered}
A^{t+\Delta t / 2}=\left(\boldsymbol{V}^{t+\Delta t}-\boldsymbol{V}^{t}\right) / \Delta t, \\
\boldsymbol{V}^{t+\Delta t}=2\left(\boldsymbol{U}^{t+\Delta t}-\boldsymbol{U}^{t}\right) / \Delta t-\boldsymbol{V}^{t}, \\
\boldsymbol{V}^{t+\Delta t / 2}=\left(\boldsymbol{U}^{t+\Delta t}-\boldsymbol{U}^{t}\right) / \Delta t, \\
\boldsymbol{U}^{t+\Delta t / 2}=\left(\boldsymbol{U}^{t+\Delta t}+\boldsymbol{U}^{t}\right) / 2,
\end{gathered}
$$

综合(7) (10)式可将(6)式改写为

$$
\begin{aligned}
& \left(\boldsymbol{M}+\boldsymbol{C} \frac{\Delta t}{2}+\boldsymbol{S}\left(\frac{\Delta t}{2}\right)^{2}\right) \boldsymbol{U}^{t+\Delta t} \\
= & \boldsymbol{F}^{t+\Delta t / 2} \frac{\Delta t^{2}}{2}+\boldsymbol{M} \boldsymbol{U}^{t}+\boldsymbol{M} \boldsymbol{V}^{t} \Delta t+\boldsymbol{C} \boldsymbol{U}^{t} \frac{\Delta t}{2}-\boldsymbol{S} \boldsymbol{U}^{t}\left(\frac{\Delta t}{2}\right)^{2} .
\end{aligned}
$$

本文采用显式求解算法 ${ }^{[15]}$, 利用有限元模拟技 术, 对该问题进行了详细的数值模拟和分析.

\section{2 编钟模型及数值模拟结果}

本文采用有限元方法模拟编钟的双音特性时, 为了突出重点, 模型主要考虑了其扁型这一主要因
素. 对钟枚等其他影响因素, 虽然它在稳定基频、抑 制噪音等方面具有重要作用 ${ }^{[1,2]}$, 但不作为本文章讨 论的重点. 另外, 对于钟体上的花纹等相关因素我们 也将在以后考虑.

图 1 是模型编钟的几何形状图, 分别是该几何造 型在 $x-y$ 平面、 $x-z$ 平面和 $y-z$ 平面上的几何投影，其中 底面椭圆形长轴长约 $0.55 \mathrm{~m}$; 短轴长约 $0.35 \mathrm{~m}$; 模型 高(钟铣长)约为 $0.4 \mathrm{~m}$; 模型横接底面长轴两端处钟 壁厚约为 $24 \mathrm{~mm}$, 短轴两端处钟壁厚约为 $15 \mathrm{~mm}$. 在 钟体其他比例因素不变的情况下, 改变钟壁的厚度, 激发出的音律基频也会随之改变 ${ }^{[4]}$, 但本文暂时不 研究钟厚变化的影响. 在用有限元方法模拟数值分 析时, 考虑编钟的材料是青铜质, 应用的一些物性参 数如下: 密度大小为 $8.9 \mathrm{~g} / \mathrm{cm}^{3}$; 泊松比为 0.25 ; 杨氏 模量为 $1.2 \times 10^{11}$; 阻尼常数为 0.02 ; 不考虑重力等因 素影响. 除钟上端部 4 个节点位移固定外, 其余边界 均为自由边界条件, 表面上正应力和剪应力恒为 0 .

图 2 是模型的切片视觉图, 可以观察到编钟的几 何模型的内腔视觉效果. 图 3 是模型的网格化结构图, 由于模型形状复杂，我们采用的是四面体网格划分， 因为四面体网格划分能够灵活有效地模拟复杂不规 则边界, 更好地模拟出编钟的真实结构. 网格密度也 是影响模拟结果的重要因素, 一般情况下, 网格间距 越小，计算精度越高，频散效应也越小，但是也会花 费更多时间和占用更大计算内存, 权衡问题的实际 情况, 此例子总节点数为 88178 个, 总单元数为 424699 个.

根据激发波在铜质材料中的传播速度 $3750 \mathrm{~m} / \mathrm{s}$, 网格化后最小网格距约为 $2.3 \mathrm{~mm}$, 由网格距与时间 步长选取之间的关系 ${ }^{[12,13]}$, 此问题模拟波传播时时 间步长 $\Delta t$ 取为 $1 \times 10^{-7} \mathrm{~s}$.

模拟敲击造成的边界力取形状近似为 Ricker 子波(图 4)脉冲型的时间函数的正值部分, Ricker 子波的一般解析表达式为

$$
f(t)=\left[1-\left(\pi f_{0} t\right)^{2}\right] \exp \left[-\left(\pi f_{0} t\right)^{2}\right],
$$
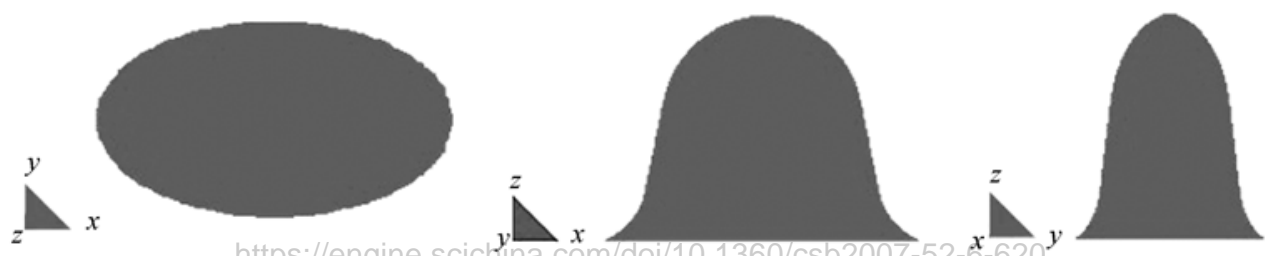

图 1 模型编钟几何模型的俯视图、正视图和侧视图 


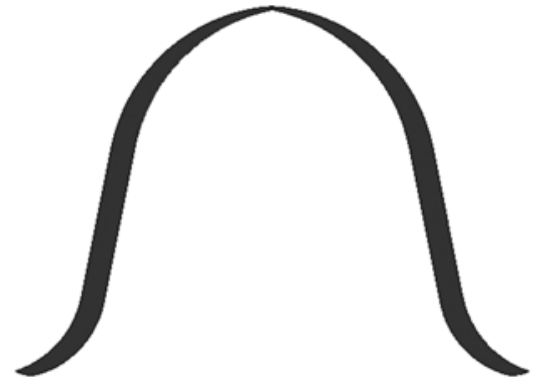

图 2 模型的切片视觉图

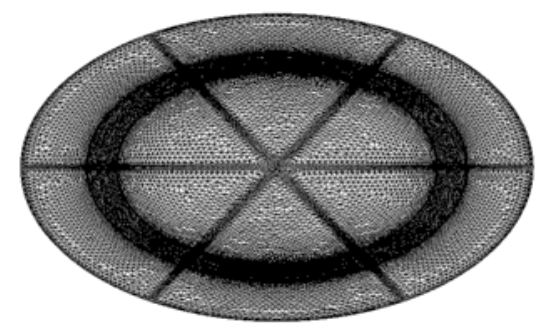

图 3 模型的网格化结构

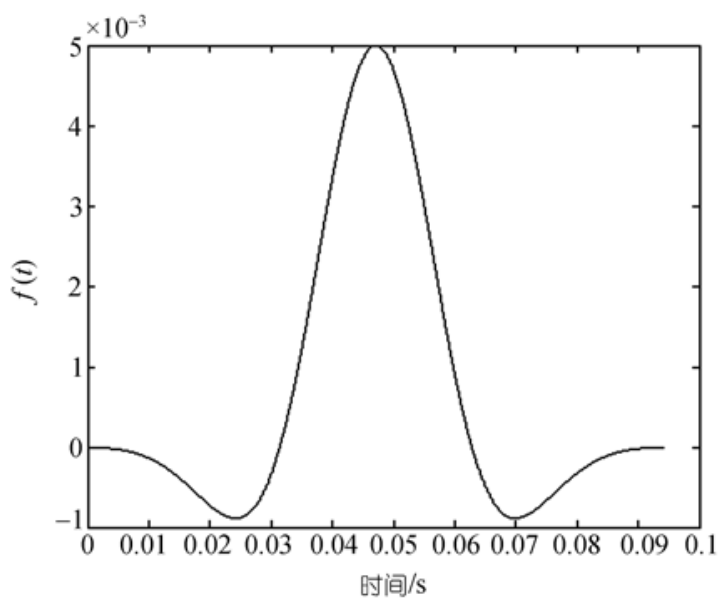

图 4 Richer 子波的波形图

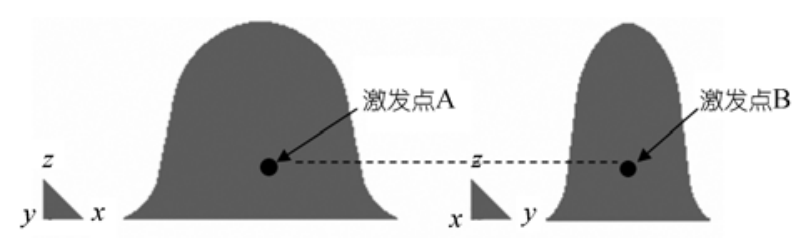

图 5 几何模型上不同的激发点正视图

其中, $f_{0}$ 为 Richer 子波的主频, 本研究中取 $f_{0}=$ $22.22 \mathrm{~Hz} .2$ 种不同的敲击位置如图 5, 2 个不同敲击点 上分别给定同样的 Richer 子波脉冲.

图 6 是在 $\mathrm{A}$ 和 $\mathrm{B}$ 两个不同点敲击激发波在扁型 钟体中传播的结果对照图. 图 6 的前 6 张图给出最初
$0.1 \mathrm{~ms}$ 的波动传播情况，可以看出敲击时波的传播， 在第 4 幅图中可以看到波在钟外壁和内壁之间多次 反射而形成的环状条纹以及波在传到下边缘时的反 射等情况; 后 4 张图显示了 $10 \mathrm{~ms}$ 过程中的几个图像, 可以看出波的传播和叠加逐步形成按特定基频为主 的振荡. 图 6 中可以明显看出, 激发位置不同, 产生 的激发波振型不同. 敲击 $\mathrm{A}$ 激发的振荡频率成分丰 富, 震荡图案花样复杂, 反映了除基频外, 其余泛音 也很丰富. 而敲击 B 激发的振荡成分较 A 相对简单, 震荡图案比较光滑, 下缘振动有起伏, 对应的是基频 的震荡. 模拟还表明这些振型是三维的, 不能简单地 以二维模型描述. 虽然我们这里没有足够的篇幅详 细展现激发的全部过程, 但不同敲击方式激发不同 三维振荡的基本物理图像已得到很清楚的显示.

图 7 是分别敲击不同点 $\mathrm{A}$ 和 $\mathrm{B}$ 时, $\mathrm{A}$ 和 $\mathrm{B}$ 处位移 分别随时间变化的时间位移波形图. 可以看出, 在同 等敲击力下, 敲击 $\mathrm{A}$ 时造成的振幅较大, 频率成分也 比较丰富; 敲击 $\mathrm{B}$ 时造成的振幅则较小, 除基频外, 其余频率成分比较单调. 二者的声波振动可以看出 有明显的不同. 图 8 是对图 7 中数据进行快速 Fourier 频谱的分析结果. 在模拟编钟激发的过程中, 编钟从 开始振动到形成共振有一时间段, 而这一时间段中 起主要作用的是 Richer 子波脉冲, 所以我们在进行 频谱分析时，不考虑这一时间段的数据而直接对编 钟形成谐振后的数据进行频谱分析. 由于人耳听觉 频率范围是 $20 \mathrm{~Hz} 20 \mathrm{kHz}$, 在模拟编钟双音特性时, 对激发出的波进行频谱分析, 我们也只给出人耳比 较敏感的频段范围的结果. 从图 8 中可以明显的看出 对于同一个模型编钟, 敲击同一个点, 在不同位置记 录到的频谱成分是不一样的; 敲击两个不同位置的 点, 在同一个位置记录到的频谱成分也是不一样的. (i ) 在敲击 $\mathrm{B}$ 时(图 8(c)和(d)), 振幅最大的是基频 $(212 \mathrm{~Hz})$; 在敲击 $\mathrm{A}$ 时(图 8(a)和(b)), 振幅最大的是 $424 \mathrm{~Hz}$ 的振荡. (ii) A 的频率成分比较丰富, 图 8 中 比较明显的峰值出现在 $212.3,424.6,530.8 \mathrm{~Hz}$, 在 $636.9,1061.6,1167.7,2017.0,2335.5,2653.9$ 和 3609.3 $\mathrm{Hz}$ 等也都存在较强的泛音. 在敲击 $\mathrm{B}$ 时, 形成的泛 音就没有那样丰富, 基频在 $212.3 \mathrm{~Hz}$, 还存在 636.9 $\mathrm{Hz}$ 的振动等, 但高频泛音总的看来不很发育. (iii) 基频为 $212.3 \mathrm{~Hz}$, 然后有 $424.6 \mathrm{~Hz}$ 的倍频, $636.9 \mathrm{~Hz}$ 位倍频等的泛音, 在物理上是合理的. (iv) A 和 B 


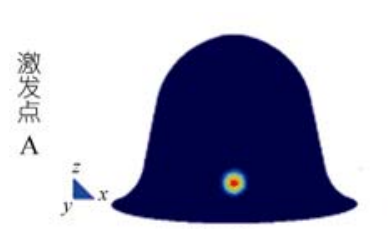

$0.00002 \mathrm{~s}$

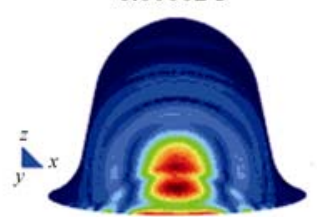

$0.0001 \mathrm{~s}$

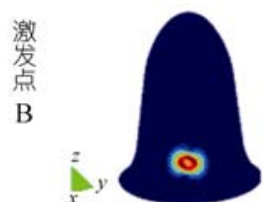

$0.00002 \mathrm{~s}$

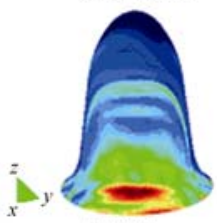

$0.0001 \mathrm{~s}$

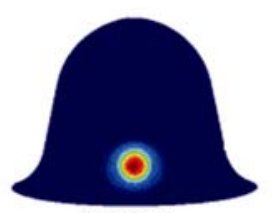

$0.00003 \mathrm{~s}$

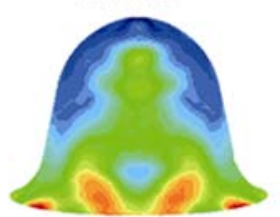

$0.0008 \mathrm{~s}$

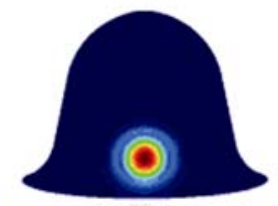

$0.0004 \mathrm{~s}$

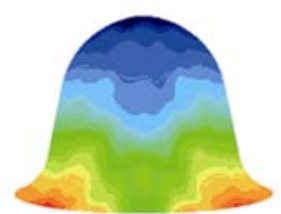

$0.004 \mathrm{~s}$

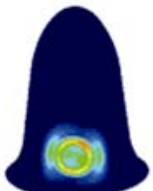

$0.00003 \mathrm{~s}$

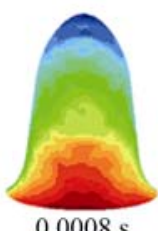

$0.0008 \mathrm{~s}$

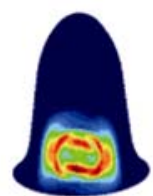

$0.00004 \mathrm{~s}$

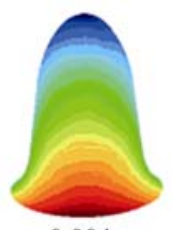

$0.004 \mathrm{~s}$

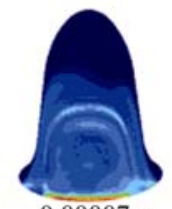

$0.00007 \mathrm{~s}$

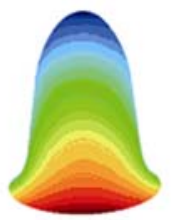

$0.0006 \mathrm{~s}$

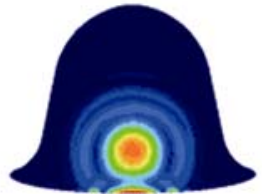

$0.00007 \mathrm{~s}$

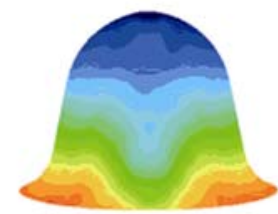

$0.006 \mathrm{~s}$
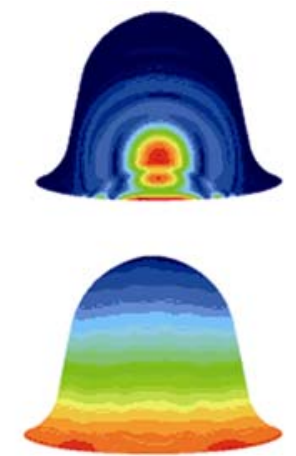

$0.01 \mathrm{~s}$

图 $6 \mathrm{~A}$ 和 $\mathrm{B}$ 两激发点在同一时刻的波动图(位移总量等值线图)对照

两种情况，有一些频率共同出现，也有一些振荡只在 $\mathrm{A}$ 中被激发或只在 B 中被激发。总之, 频谱分析清楚 显示了不同激发方式下的双音特性.

\section{3 讨论}

首先, 本文的初步计算显示了用有限单元法数 值模拟曾侯乙编钟在不同敲击方式下双音的激发过 程是可行的. 振动图案的时间变化和比较、振动曲线 的比较和频谱分析结果, 均显示了数值计算可以用 于分析不同敲击方式激发双音的物理过程，使我们 可以对解释双音之谜提供一条定量化的、可视化的新 途径. 敲击 $\mathrm{A}$ 处时, 由于该处曲率小、刚度小、易变 形, 因此振动频率丰富, $424.6 \mathrm{~Hz}$ 的频率成为主要成 分; 敲击 B 处时, 由于该处曲率大、刚度大、不易变 形, 因此振动频率比较单调, $212.3 \mathrm{~Hz}$ 为主要成分. 这些计算结果在物理上是合理的. 计算的振动频率、 $\mathrm{A}$ 和 $\mathrm{B}$ 存在共同成分, 也存在不同成分, 特别是基频 $212.3 \mathrm{~Hz}$ 及其倍频 $424.6 \mathrm{~Hz}$ 在 A 中的出现和占主导 地位、其三倍频在 B 中的出现等, 定量地进一步显示 了数值计算模拟结果的合理性.
尽管我们没有足够的篇幅来详细讨论振荡的激 发过程, 但可以看出, 钟的振荡基频和主要低频振荡 在一定时间后与初始敲击的 Richer 子波频率无关， 主要与弹性波的传播、反射和叠加、干涉有关. 编钟 存在一定的振荡模式, 在敲击不同点时, 有时形成相 长干涉，一些振型得以发育; 有时形成相消干涉，一 些振型被压制.

真正的曾侯乙编钟, 其取材要求严格、钟腔结构 复杂，才保证其高质量的音乐特性. 本文采用有限元 方法模拟编钟的双音特性时, 模型主要考虑了其横 截面是椭圆形这一主要因素，探讨了不同激发点的 影响. 而对频率特性与钟体的材料、精细几何形状、 铣长、装饰图案等关系, 尚未展开研究. 我们的模拟 也仅对一个钟体进行了分析, 如何尺寸和造型才能 使整套编钟成为和谐的乐器, 也需要做大量的研究. 我们的祖先以惊人的聪明才智、设计和制造了曾侯乙 编钟，甚至被称为“世界第八奇迹”，我们今天也应该 能够运用先进的计算技术，进一步揭开编钟的奥秘.

数值模拟分析结果表明, 编钟的双音特性主要 

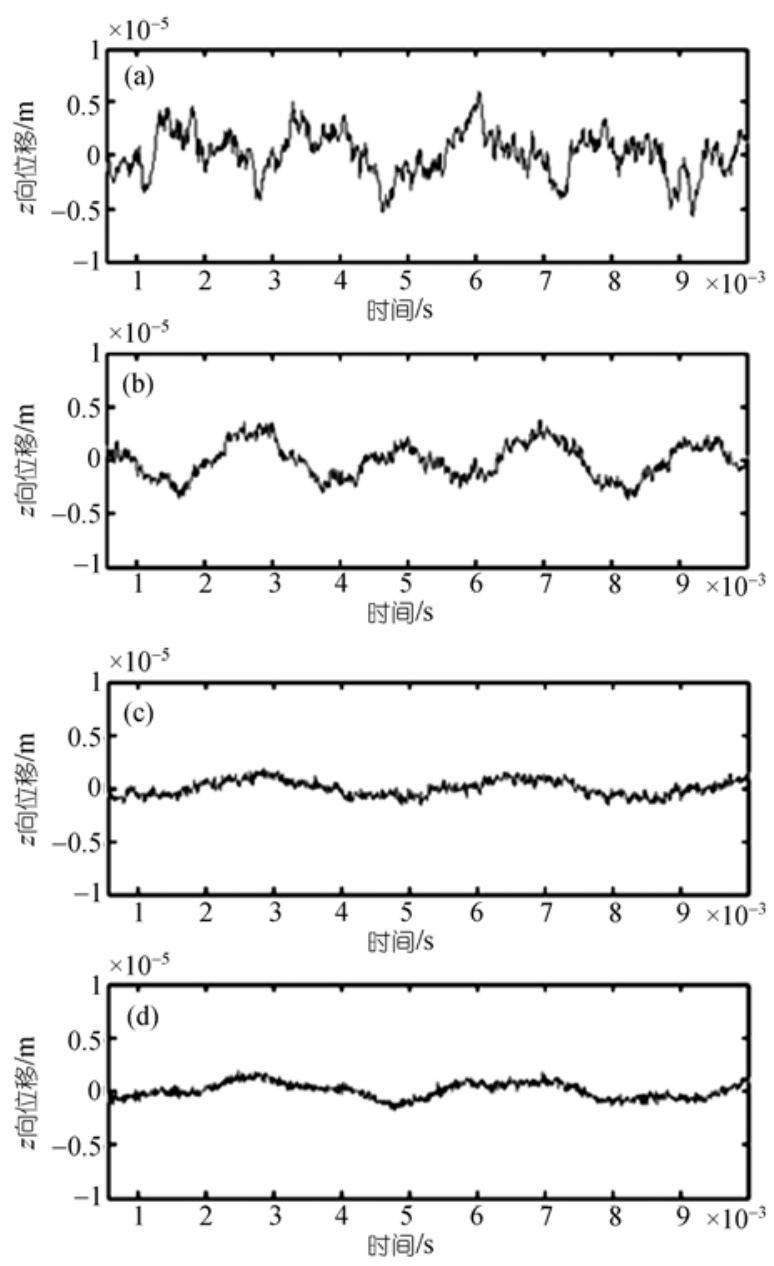

图 7

(a) 敲击 $\mathrm{A}$ 点时 $\mathrm{A}$ 的振动图; (b) 敲击 $\mathrm{A}$ 点时 $\mathrm{B}$ 的振动图; (c) 敲击 $\mathrm{B}$ 点时 A 的振动图; (d) 敲击 B 点时 B 的振动图

由编钟的几何形状和激发部位来控制. 不同科学领 域内有许多问题在实质上是相通的. 我们知道, 特大 地震, 如 1960 年的智利大地震及 2004 年 12 月 26 日 印度尼西亚苏门答腊大地震, 都激发了地球的自由 振荡, 不同的地震激发的地球自由振荡情况不同、同 一地震在地球不同部位造成的振荡也不相同. 本文 的方法已经显示了敲击在不同部位引起的振荡位移 是不同的, 原则上也可以运用于大地震激发的横向 不均匀地球内部自由振荡激发问题高精度的数值模 拟, 从而对地球在地震触发下自由振荡规律及其影 响的这一重要科学问题做更深入的解释.

由于模拟过程中各种条件的限制对模拟结果都 有一定的影响, 网格单元数及计算时间长度的限制, 都会给模拟结果带来一定的误差. 初步计算已经表 明利用有限单元法计算波的传播和激发自由振荡过
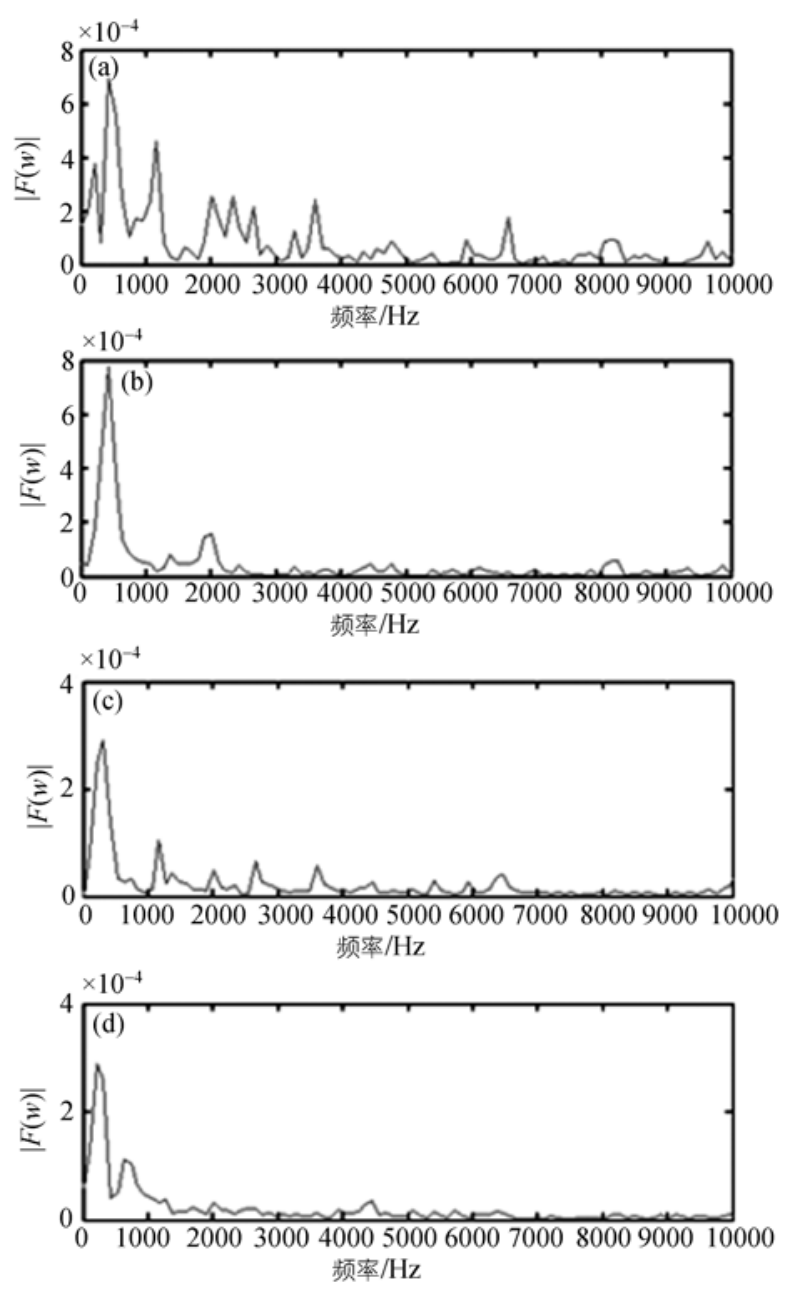

图 8 敲击时激发的振动的频谱分析结果

(a) 敲击 A 点时 A 点振动的频谱分析; (b) 敲击 A 点时 B 点振动的频 谱分析; (c) 敲击 B 点时 A 点振动的频谱分析; (d) 敲击 B 点时 B 点振 动的频谱分析

程的科学思路是可行的, 今后如果模拟真正复杂形 状的编钟, 需要开展更大规模的并行有限元计算 [16,17]. 在并行计算中采用区域分解法, 首先将整个物 理模型分解为若干不相重叠的子区域, 相应的有限 元模型也根据所选并行算法做离散化处理, 将每个 子区域模型独立分配给一个计算进程处理, 并行地 进行单元计算, 分布式总体刚度矩阵合成计算和并 行大规模线性系统的求解. 而各个子区域之间的内 边界黏结条件则借助于针对于不同并行算法相应的 计算进程之间信息交换与迭代求解来完成. 目前, 我 们已经完成了并行程序的调试, 即将在今后进一步 研究工作中得以应用.

\section{4 结论}

本文基于弹性波动理论用有限单元法进行数值 
计算, 模拟了仿曾侯乙编钟模型在不同敲击部位下 振荡的激发过程，通过对敲击不同部位时激发波动 的传播图案、对钟体质点振动位移的时间变化记录和 对振荡曲线的频谱分析等多方面分析, 有效地显示 了曾侯乙编钟不同的敲击部位产生了 2 种不同频率 特性的乐音的物理过程以及编钟的双音特性通过相 长与相消干涉的实现.

曾侯乙编钟让我们不得不折服于我国古代先人 的聪明与智慧, 它吸引了无数的音乐爱好者和科学 爱好者对它的研究兴趣. 到目前为止, 我们对此编钟 的研究远未结束, 它极其复杂的内腔结构需要我们 更深层地认识和进一步的研究工作. 另外, 我们还将 对钟体的材料组分、厚度、内部的复杂结构及钟体表 面上的钟枚、花纹等相关因素继续进行研究分析. 我 们相信, 各个学科的综合努力将有助于进一步揭开 曾侯乙编钟的一系列难解之谜.

致谢 感谢 Y. Ricard 的帮助和两位审稿人的建设性意见.

$$
\text { 参考文献 }
$$

1 严燕来, 孔令达, 柴康敏, 等. 中华古编钟的乐钟性质研究. 大 学物理, 2004, 23(2): 53-57

2 韩宝强. 双音钟音乐效能之检测. 乐器, 2002, 7: 18-22

3 华觉明. 从陶寺铜铃到中华和钟——双音青铜编钟的由来、衍变 和创新. 清华大学学报, 2000, 15(5): 72-79
4 冯光生. 周代编钟的双音技术及应用. 中国音乐学, 2002, 1: 4054

5 王大钧, 陈健, 王慧君. 中国乐钟的双音特性. 力学与实践. 2003, 25(4): 12-16

6 张永刚. 地震波场数值模拟方法. 石油勘探. 2003，42(2): 143148

7 王县成. 有限单元法. 北京: 清华大学出版社, 2003. 14-253

8 Li X, Zhou H. Partition of unity finite element method for short wave propagation in solids. Appl Math Mech, 2005, 26(8): 10561063[DOI]

9 Liu Y, Qiao A, Feuchter D, et al. Finite element method on numerical simulation of stratum corneum's penetration property. Appl Math Mech, 2006, 27(4): 469-475[DOI]

10 王琥，李光耀，钟志华. 有限元并行计算中网格自动分区的优化. 工程力学, 2005, 22(增刊): 46-51.

11 胡德绥. 弹性波动力学. 北京: 地质出版社, 1989. 1-122

12 Komatitsch D, Tromp J. Introduction to the spectral element method for three-dimensional seismic wave propagation. Geophys J Int, 1999, 139: 806-822[DOI]

13 Komatitsch D, Tromp J. Spectral-element simulations of global seismic wave propagation- I. Geophys J Int, 1999, 149: 390412[DOI]

14 Thompson L L, Huan R. Finite element formulation of exact non-reflecting boundary conditons for the time-dependent wave equation. Int J Numer Meth Eng, 1999, 45: 1607-1630[DOI]

15 寇哲君, 程建钢, 黄文彬, 等. 结构动力分析显隐式混合积分并 行算法及实现. 清华大学学报(自然科学版), 1999, 39(4): 21-24

16 刘耀儒, 周维恒, 杨强, 等. 三维有限元并行计算及其工程应用. 岩石力学与工程学报, 2005, 24(14): 2434-2438

17 陈国良. 并行计算——结构·算法·编程. 北京: 高等教育出版社, 2003. 1-280 